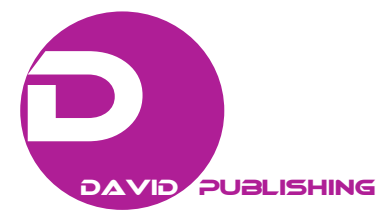

\title{
Primary Liver Tuberculosis Presented as Acute Alithiasis Cholecystitis
}

\author{
Hamri Asma, Narjis Youssef and Benelkhaiat Ridouan \\ Department of Surgery, Ibn Tofail Hospital, MohamedVI University Hospital, University of Medicine and Pharmacy, Marrakech \\ 40000, Morocco
}

\begin{abstract}
Isolated liver tuberculosis is still considered a rare condition and atypical clinical presentation challenges the clinical acumen of the treating physician. Hepatic tuberculosis is usually associated with an active pulmonary or miliary tuberculosis. Liver involvement in tuberculosis is usually clinically silent. A lady presented with fever, pain in right hypochondria, radiate to the right shoulder or scapula nausea and weight loss. CT scan of abdomen showed acute cholecystitis and multiple small hypodense non-enhancing lesions and a heterogeneous texture of liver. Biopsy confirmed the diagnosis of hepatic tuberculosis. It was concluded a case of isolated hepatic tuberculosis without evidence of other primary sites involvement. It is important to consider tuberculosis in the differential diagnosis when suspecting metastatic diseases in a patient with vague symptoms and abnormal hepatic texture on CT.
\end{abstract}

Key words: Hepatic tuberculosis, caseating granuloma, antitubercular therapy.

\section{Background}

Tuberculosis (TB) remains an important public health problem in Morocco. Liver involvement in tuberculosis is usually clinically silent. Occasionally, local signs and symptoms may be prominent in hepatic tuberculosis, and may constitute the initial or sole presenting feature of the disease. However, even in developing countries, liver tuberculosis accompanied by local symptoms is an uncommon entity [1].

\section{Case Report}

A 50 year-old woman, she has admitted to surgical ward with right upperabdominal pain. There was no history of exposure to tuberculosis. The patient was well and the vital signs were stable. Physical examination showed no palpebral bulbar conjontiva jaundice, $38^{\circ} \mathrm{C}$ of temperature, blood pressure $120 / 90$ mmhg, respiratory rate was 20 breaths/min and his heats rate was 89 beats/min. right hypochondrial pain, Murphy sign positif; No abnormality was noted by chest percussion and there was no lymphadenopathy.

Corresponding author: Benelkhaiat Ridouan, Ph.D., research field: surgery.
Laboratory data revealed an elevated $\mathrm{C}$ reactive protein (CRP) level of 152 and hyperleukocytosis, normal liver and renal function tests. Abdominal ultrasound and abdominal scan CT revealed a cholecystitis with non specific-nodular lesion in the I subsegment of the liver measuring $1 \mathrm{~cm}$. No ascites or adenopathy was seen. The Chest X-Ray was normal. The patient was operated 48 hours later; surgical exploration: cholecystitis (alithiasic) with nodule (I subsegment of the liver). Nodule biopsy and cholecystectomy was done. Histological study suggested an inflammatory granuloma pseudotumor with Langerhans giant cells and caseous necrosis. Anti-TB medications including isoniazid, rifampin, ethambutol, and pyrazinamide were administered accordingly. The patient completed the 6-month course of medication without severe adverse events. Follow-up was continued for two years, and the patient is still healthy.

\section{Discussion}

There are three forms of hepatic tuberculosis. Diffuse hepatic involvement with pulmonary or miliary tuberculosis is the most common form seen in $50 \%$ to 
$80 \%$ of patients dying of pulmonary tuberculosis. Diffuse hepatic infiltration without recognizable pulmonary involvement is the second form. The third very rare form presents as a focal/local tuberculoma or abscess. Isolated Liver Tuberculosis is the rarest form of local hepatic tuberculosis [2]. Kok et al. [3] reported an overall incidence of $0.3 \%$ for isolated hepatic tuberculosis. Hepatic tuberculosis lesions that appear as masses larger than $2 \mathrm{~mm}$ in diameter are referred to as macronodular and pseudotumoural tuberculosis. On the basis of imaging examinations alone, these lesions are virtually indistinguishable from many other focal lesions of the liver, such as hepatocellular carcinoma, metastases and Hodgkin's disease, so pathological examination is necessary for diagnosis. The difficulty is reaching a correct preoperative diagnosis of nodular hepatic tuberculosis that presents as a spaceoccupying lesion. It is usually unsuspected and confused with primary or metastatic carcinoma of the liver. The radiologic diagnosis of hepatic tuberculoma is difficult. The histological picture of hepatic tuberculoma is usually that of a large epitehloid tumour composed of conglomerate tubercles with central caseation necrosis [4]. Langerhans-type gaint cells may be found in the granuloma and are surrounded by lymphohistiocytic cells, plasma cells and eosinophils [5]. In view of the nonspecific presentation and imaging appearance of the disease, a high index of suspicion is required to obtain a preoperative diagnosis [6]. In this case, the diagnosis was established at laparotomy or laparoscopy. The importance of establishing the correct diagnosis cannot be overstated, since untreated abdominal tuberculosis carries a 50\% mortality rate.

\section{Conclusion}

Tuberculosis should be kept on the differential diagnosis of multiple lesions in liver, especially in endemic areas. Laboratory tests and imaging can be challenging and should be analyzed thoroughly supplementing with other data where necessary.

\section{References}

[1] Bangroo, A. K., and Malhotra, A. S. 2005. "Isolated Hepatic Tuberculosis." JIAPS 10 (2): 105-7.

[2] Purl, A. S., Nayyar, A. K., and Vij, J. C. 1994. "Hepatic Tuberculosis.” Indian Journal Tub. 41 (3): 131-4.

[3] Kok, K. Y., and Yapp, S. K. 1999. "Isolated Hepatic Tuberculosis: Report of Five Cases and Review of the Literature." J. Hepatobiliary Pancreat Surg. 6 (2): 195-8.

[4] Ruksana, K., Chowdhury, Md. M., and Khan, A. S. 2014. “A Case Report: Isolated Liver Tuberculosis.” BSMMU 7 (1): 71-3.

[5] Tan, T. C., Cheung, A. Y., Wan, W. Y., and Chen, T. C. 1997. "Tuberculoma of the Liver Presenting As a Hyperechoic Mass on Ultrasound.” Br. J. Radiol. 70 (840): 1293-5.

[6] Debnath, P. R., Tripathi, R., Kandpall, D., Kumar, B., Malik, E., and Sharma, S. B. 2007. "Isolated Tubercular Liverabscess in Children Treated with Percutaneous Isoniazid Infusion." Indian J. Tuberc. 54 (3): 149-51. 